\title{
Preparation of one-component addition-cure liquid silicone rubber coating with enhanced storage stability and bond strength
}

\section{Chaohua Li}

Hunan normal university

Shengpei Su ( $\square$ shengpei_academi@sohu.com )

Hunan Normal University https://orcid.org/0000-0003-4225-1468

\section{Bikui Wang}

Hunan Normal University

Jijia Zhou

Hunan Normal University

\section{Research Article}

Keywords: OLSR coating, storage stability, printed circuit boards, bond strength, salt spray resistance

Posted Date: June 29th, 2021

DOl: https://doi.org/10.21203/rs.3.rs-197037/v1

License: (c) This work is licensed under a Creative Commons Attribution 4.0 International License.

Read Full License

Version of Record: A version of this preprint was published at Journal of Materials Science: Materials in Electronics on July 23rd, 2021. See the published version at https://doi.org/10.1007/s10854-021-065979. 


\section{Abstract}

It is of great interest and challenge to simultaneously improve the storage stability and bond strength of one-component addition-cure liquid silicone rubber (OLSR) coating for electronic circuit board. In this work, we proposed an efficient approach to address this issue by incorporating both cyano-modification platinum catalyst (CN-PT) and urethane-containing poly(hydromethylsiloxane) (N-PHMS) into OLSR coating using a mechanical mixer. The CN-PT and N-PHMS were synthesized through thermal polymerization reaction and hydrosilylation reaction, respectively. The OLSR coating containing $0.3 \mathrm{phr}$ $\mathrm{CN}-\mathrm{PT}$ and $3 \mathrm{phr} \mathrm{N}-\mathrm{PHMS}$ showed the best properties, with a tensile bond strength of $1.63 \mathrm{MPa}$, a storage period $>180 \mathrm{~d}$ at $25^{\circ} \mathrm{C}$, and a curing rate $<2 \mathrm{~h}$ at $80^{\circ} \mathrm{C}$. The salt-spray resistance and continuous power-on performance of OLSR coating were also improved, compared with pure OLSR coating. The synergism of $\mathrm{CN}-\mathrm{Pt}$ and N-PHMS on the enhancement both the storage stability and bond strength of OLSR coating was also explored. Our findings exhibited great potentials for fabricating OLSR coating with excellent long-term stable service performance including high storage stability and protective ability.

\section{Introduction}

Printed circuit boards (PCBs) are the heart of the communication and electronics industry. Most of time, PCBs are often exposed to humidity leading to the corrosion of printed circuit boards and subsequent failure of the electronic devices[1-5]. Currently, electrically conductive adhesives[6], nanosized Ag paste[7] and carbon nanotubes[8] are the most commonly used packaging materials in the electronics industry. However, traditional packaging materials are not suitable for modern electronic packaging of printed circuit boards because of high conductivity and cost. In general, silicone rubbers coating is widely used in protection of PCBs because of its good dielectric properties, water repellency, ultraviolet durability, excellent chemicals and thermal degradation resistance $[9,10,11]$. Nowadays, solvent-based condensation-cure silicone rubber coating [12] for the protection of PCBs is one of the few commercialized coatings. During the curing process of solvent-based condensation-cure silicone coating, the solvents and small molecules will be released leading to the pollution of the environment and the PCB[13], even the reduction of the reliability and the service life of electronic products.

One-component addition-cure silicone rubber (OLSR) coating has the advantages of low shrinkage, the ability to adjust reaction speed and conditions over a wide range, and the fact that no byproducts or degradation products are formed[14-18]. It can obviously solve the problem caused by the released solvents and small molecules for solvent-based condensation-cure silicone rubber. Although the utility of OLSR coating was accepted, it was not often well satisfied with their applications in the fields of the protection of PCBs due to its storage instability and poor bond strength. Therefore, how to improve the storage stability and bond strength of OLSR coating has become an important direction for the researchers in the field of protection of PCBs.

Thermal-latent hydrosilylation catalyst is crucial for the enhancement of the storage stability of OLSR coating. A typical series of thermal-latent hydrosilylation catalysts consisting of $\mathrm{H}_{2} \mathrm{PtCl}_{6}$ and special 
polymers was developed by Katsuhiko research group[19]. These catalysts were successfully applied in the curing process of silicone resin with high thermal-latent characteristics. Many functional groups including propargyl[20], amine[21], 1-phenylethylisocyanide[22], isocyanide[23] often caused the poison of the platinum catalysts. However, most of these thermal-latent hydrosilylation catalysts are used at a high curing temperature for addition-cure liquid silicone rubber (the curing temperature $>100{ }^{\circ} \mathrm{C}$ ), and a low storage temperature $\left(<10^{\circ} \mathrm{C}\right)$. They are not suitable for OLSR coating obviously.

For enhancing the bond strength of OLSR coating, an effective way is to incorporate an adhesion promoter on OLSR coating to make it self-adhering when cured on the surface of the substrate. There are a lot of reports devoted to the studies of adhesion promoters. Examples include organosilanes with silylene group as adhesion promoters to adhere silicone elastomers on polyethylene[24]; organosilanes with epoxy group enhancing the adhesion on polyethylene terephthalate[25, 26, 27]; organosilanes with phenyl group and epoxy group enhancing the adhesion on electrolyte membrane[28]; organosilanes with ester groups and vinyl groups enhancing the adhesion on polycarbonate[29, 30]; organosilanes with tetratert-butyl titanate enhancing the adhesion on copper, polybutylene terephthalate, polyphenylene sulfide and polystyrene[31]; organosilanes with allyl group enhancing the adhesion to nylon[32]; organosilanes with isocyanurate group enhancing the adhesion on epoxy resin, phenolic resin[33]. However, there are few reports on the addition of adhesion promoter on the addition-cure silicone rubber to enhance adhesion to PCBs.

In this work, cyano groups will be introduced to platinum catalyst (CN-PT) for enhancing the storage stability, and urethane-containing poly(hydromethylsiloxane) (N-PHMS) will be synthesized through hydrosilylation as an adhesion promoter for enhancing the bond strength. Fourier transform infrared spectroscopy (FT-IR) will be used to characterize the chemical structure of CN-PT and N-PHMS.The mechanical properties and curing rate of OLSR coating will be tested in order to obtain optimum content of N-PHMS and CN-PT. The properties including storage stability, bond strength, surface morphology, protective performance of the OLSR coating will be tested to evaluate the advantages of this combination designed OLSR coating for PCBs.

\section{Experimental Section}

\subsection{Materials}

Acrylonitrile (AN) and hydroxyethyl acrylate (HEA) were purchased from Evonik Industries AG, Germany and Aladdin Co., Ltd., China, respectively. The ethynylcyclohexano platinum catalyst (platinum content was 5000 ppm) was supplied by Shin-Etsu Co., Ltd, Japan, and 3-isocyanatopropyltriethoxysilane (ICPES) was provided by JiangsuHuasheng Fine Chemical Co., Ltd., China. Poly(hydromethylsiloxane) -1(PHMS-1, 0.1 wt\% hydride), poly(hydromethylsiloxane)-2 (PHMS-2, 0.5 wt\% hydride), MQ silicone resin (MQ, $2 \mathrm{wt} \%$ vinyl) and vinylterminated poly(dimethylsiloxanes) (VPDMS, $0.28 \mathrm{wt} \%$ vinyl) were obtained from Elkem Silicones Co., Ltd., China. TL-GC02 silicone coating was purchased from Chengdu Taly Technology Co.,Itd, China. 


\subsection{Synthesis of the cyano-containing platinum catalyst (CN-PT)}

CN-PT was synthesized based on a one-step thermal polymerization reaction[21], as shown in Fig. 1. This reaction was achieved by mixing $100 \mathrm{~g}$ of the commercially bought platinum catalyst (Pt) with $200 \mathrm{~g}$ of ethyl acetate in a 500-mL four-necked round-bottomed flask equipped with a mechanical stirrer. The mixture was stirred for $0.5 \mathrm{~h}$ at $60^{\circ} \mathrm{C}$, and then acrylonitrile (AN, $2 \mathrm{~g}, 4 \mathrm{~g}, 6 \mathrm{~g}, 8 \mathrm{~g}, 10 \mathrm{~g}$ ) were added dropwise. Thereafter, the reaction mixture was stirred for another $6 \mathrm{~h}$ at $80^{\circ} \mathrm{C}$ before being distilled under vacuum in order to obtain the cyano-containing platinum catalyst (CN-PT). Figure 4 shows the FT-IR spectra of AN, Pt, and CN-PT.

\subsection{Synthesis of urethane-containing poly(hydromethylsiloxane) (N-PHMS)}

The two-step hydrosilylation reaction developed herein was used to synthesize the N-PHMS adhesion promoter. First, hydroxyethyl acrylate (HEA, $10 \mathrm{~g})$, ethyl acetate $(200 \mathrm{~g})$, and the platinum catalyst $(0.2 \mathrm{~g})$ were mixed in a $500-\mathrm{mL}$ four-necked round-bottomed flask for $0.5 \mathrm{~h}$ at $60^{\circ} \mathrm{C}$. Subsequently, poly (hydromethylsiloxane) (PHMS-1, $100 \mathrm{~g}$ ) was added dropwise to the mixture, followed by stirring for $3 \mathrm{~h}$ at $60^{\circ} \mathrm{C}$ and then heating to $90^{\circ} \mathrm{C}(3 \mathrm{~h})$. Afterwards, 3-isocyanatopropyltriethoxysilane (ICPTES, $21.3 \mathrm{~g}$ ) was added to the flask in a dropwise manner, and the reaction was continued for another $3 \mathrm{~h}$. Finally, reduced pressure distillation was applied to remove ethyl acetate and isolate the synthesized N-PHMS product. The synthetic scheme is shown in Fig. 2, and Fig. 5 presents the FT-IR spectra of PHMS and N-PHMS.

\subsection{Preparation of the OLSR samples}

VPDMS, PHMS-2, CN-PT, N-PHMS, 1-ethynylcyclohexanol, and Karstedt's catalyst were uniformly mixed using an electric mixer, and then they were vulcanized at the appropriate temperature in order to obtain the OLSR coating. CN-PT and N-PHMS were uniformly dispersed in the OLSR coating. The proportions of materials used to prepare different samples of OLSR coating are listed in Table 1. 
Table 1

Components and proportions used to prepare different OLSR

samples.

\begin{tabular}{|llllll|}
\cline { 2 - 4 } Component (mass, g) & OLSR-1 & OLSR-2 & OLSR-3 & OLSR-4 \\
\hline VPDMS & 100 & 100 & 100 & 100 \\
PHMS & 5 & 5 & 5 & 5 \\
MQ resin & 20 & 20 & 20 & 20 \\
CN-PT & - & 0.3 & - & 0.3 \\
N-PHMS & - & - & 3 & 3 \\
1-Ethynylcyclohexanol & 0.1 & 0.1 & 0.1 & 0.1 \\
Karstedt's catalyst & 0.3 & - & 0.3 & - \\
\hline
\end{tabular}

\subsection{Characterization}

\subsubsection{Fourier transform infrared(FT-IR) spectrometry}

Liquid OLSR samples were coated on the surface of $\mathrm{KBr}$ slice for IR absorption measurement using an Avatar 370 FT-IR spectrometer (Thermo Nicolet, American). The wavelength range, resolution, and number of scans were set to $4000-400 \mathrm{~cm}^{-1}, 4 \mathrm{~cm}^{-1}$, and 16 , respectively.

\subsubsection{Mechanical properties}

The tensile strengths of the OLSR coatings were measured by using a universal testing machine (HDWDW-100A, Xiamen HaiDa Inc., China), according to ISO 37-2005. The Shore A hardness of the samples was assessed using a Shore A durometer (LX-A, Shanghai Liuling Instruments Factory, China), in accordance with ISO 7619-1-2004.

\subsubsection{Tensile bond strength testing}

The tensile bond strengths of the OLSR coatings were measured by using a universal testing machine (HD-WDW-100A, Xiamen HaiDa Inc., China), according to ASTM D 3164-03 protocols, respectively.

\subsubsection{Time-viscosity testing}

The viscosity of different OLSR coating samples was monitored throughout the curing process in order to examine the curing rate. The measurements were performed using a rotating viscometer (NDJ-8S, Shanghai Jitai Inc., China), in accordance with ASTM-D 1084-16.

\subsubsection{Surface morphology}

After tensile shear failure, the morphology of the failure surface was analyzed by field emission-scanning electron microscopy (FE-SEM, EVO-18, Carl Zeiss AG, Germany) and energy dispersive spectrometer (EDS, 
Oxford Inca 250, Oxford Instruments Co., Britain). The surface tension of the OLSR samples was measured using a Spinning Drop Interface Tensiometer (TX500, Kino, China).

\subsubsection{Salt-spray testing}

Salt-spray tests were performed on PCBs coated with OLSR coating (300 h), according to the ASTMB 117 protocol.

\subsubsection{Continuous power-on testing}

Continuous power-on tests were carried out on samples of bluetooth headset circuit boards coated with OLSR coating, as shown in Fig. 3. Brine was used as liquid medium, and the time of continuous power-on of the PCBs was recorded.

\section{Results And Discussion}

\subsection{ATR-FTIR analysis}

\subsubsection{CN-PT}

The cyanide group in acrylonitrile was grafted onto the platinum catalyst in order to improve the storage stability of the OLSR coating material. To ensure that the grafting process was successful, the CNmodified catalyst was characterized by attenuated total reflectance-Fourier transform infrared (ATR-FTIR) spectroscopy in the range of 400-4000 cm-1. The CN-PT spectrum illustrated in Fig. 4 shows absorption peaks at 1715 and $1625 \mathrm{~cm}-1$ corresponding to the vibrations of carbonyl and C-N (primary amide) groups, respectively. The 3074 and $2232 \mathrm{~cm}-1$ peaks observed in the spectrum of acrylonitrile are characteristic of the vinyl and nitrile groups, respectively, and they are not evident in the CN-PT spectrum. These results indicate that nitrile was successfully transformed to a primary amide by hydrolysis reaction, and that the primary amide is well grafted onto the platinum catalyst by vinyl addition reaction. Thus, the storage stability of the OLSR produced using the CN-PT catalyst is expected to be improved.

\subsubsection{N-PHMS}

Addition-cure silicone is nonpolar in nature. Therefore, to enhance the adhesion of this material on PCBs, the hydromethylsiloxane used in the curing process was grafted with ICPTES. The modified (N-PHMS) and non-modified (PHMS) hydromethylsiloxane samples were characterized by ATR-FTIR in the range of 400-4000 cm-1. N-PHMS presents peaks at 1706, 1524, and $1363 \mathrm{~cm}-1$ corresponding to carbonyl, N-H bond, and $\mathrm{C}-\mathrm{N}$ bond vibrations in a secondary amide, respectively. These peaks are not evident in the PHMS spectrum. Meanwhile, the characteristic peaks of HEA (3600-3200 cm - 1) was not found. Thus, we may conclude that HEA was successfully grafted with PHMS by hydrosilylation reaction. And besides, the hydroxyl was successfully transformed to secondary amide by reaction.

\subsection{Effect of N-PHMS on the mechanical properties of OLSR}


The effect of N-PHMS on the mechanical properties of OLSR coating, namely, tensile strength and Shore A hardness, was analyzed. The obtained results illustrated in Fig. 6 show that neat OLSR has a tensile strength of about $1.83 \mathrm{MPa}$. Increasing the content of N-PHMS in the OLSR coating increases its tensile strength up to $2.87 \mathrm{MPa}$ at $3 \% \mathrm{~N}-\mathrm{PHMS}$. Further increase in N-PHMS content results in reduced tensile strength, with the $6 \% \mathrm{~N}$-PHMS OLSR sample yielding a value of $1.63 \mathrm{MPa}$. Meanwhile, Shore A hardness decreases continuously with increasing N-PHMS content, and it reaches 15 in the $6 \%$ N-PHMS OLSR sample. To explain these trends, we consider that the Si-H groups in N-PHMS may undergo addition reaction with VPDMS during the curing process (Fig. 7). This results in the formation of an interpenetrating network structure that is closely packed and thus, has a larger crosslinking density, compared with the original material. Within a certain range of cross-linking density values, tensile strength increase strongly with increasing N-PHMS content. However, when the density exceeds a certain limit, the tensile strength begins to decrease. But the Shore A hardness continues to decrease with increasing N-PHMS content, due to the introduction of more branches. Overall, the results demonstrate that the optimum N-PHMS content is $3 \%$.

\subsection{Effect of CN-PT on the curing rate of OLSR coating}

To evaluate the activities of CN-PT catalysts synthesized using different concentrations of acrylonitrile, the viscosity of the OLSR coatings prepared using these catalysts was monitored throughout the hydrosilylation process in order to assess the efficiency of VPDMS and PHMS conversion. The initial ( $0 \%$ conversion) and final (nearly $100 \%$ conversion) viscosity values of the reaction mixture are 1000 and $100000 \mathrm{cps}$, respectively. Since the conventional storage and curing temperatures of OLSR coating are 25 and $80^{\circ} \mathrm{C}$, the evolution of viscosity as a function of time was monitored at these temperatures. The results depicted in Figs. 8 and 9 demonstrate that the curing rate of OLSR coating decreases with increasing acrylonitrile concentration (2-10\%), irrespective of temperature. At $25^{\circ} \mathrm{C}$ and acrylonitrile concentration $\geq 6 \%$, OLSR coating could not be cured, even after 180 days (Fig. 8 ). However, at $80^{\circ} \mathrm{C}$, the OLSR coating is cured within only $2 \mathrm{~h}$, using CN-PT catalysts prepared with acrylonitrile concentrations $\leq$ $6 \%$ (Fig. 9). These results can be explained based on the electron adsorption theory. Briefly, when the electrons in the $\pi$ bond of the amide group enter the $d$ orbital of Pt, a strong adsorption bond is formed between acrylonitrile and the platinum catalyst. This bond inhibits the efficiency of the hydrosilylation process, thereby suppressing the curing rate of OLSR. At elevated temperatures $\left(80^{\circ} \mathrm{C}\right)$, the adsorption bond dissociates, and the catalytic rate of hydrosilylation increases. Overall, our results demonstrate that the activity of CN-PT catalysts may be controlled simply by varying the concentration of acrylonitrile (higher concentrations lead to lower curing rates), which is consistent with previously reported data[18]. Considering that the concentration of $6 \%$ affords both, a long storage period $\left(>180\right.$ days at $25^{\circ} \mathrm{C}$ ) and a high curing rate (cured within $2 \mathrm{~h}$ at $80^{\circ} \mathrm{C}$ ) of OLSR coating, it is taken to be the optimum concentration of acrylonitrile.

\subsection{Properties of different OLSR samples}

Four samples of OLSR coating were prepared in this study using different proportions of starting materials, as show in Table 1. The properties of these samples were analyzed and compared, as 
discussed below.

\subsubsection{Curing rate analysis}

As shown in Figs. 10 and 11, the curing rates of OLSR coatings decrease in the following order: OLSR-1 > OLSR-3 $>$ OLSR-2 $>$ OLSR-4 for both 25 and $80^{\circ} \mathrm{C}$ conditions. The availability of the amide $\mathrm{N}$ element in OLSR-2 (CN-PT), OLSR-3 (N-PHMS), and OLSR-4 (CN-PT and N-PHMS) suppresses the curing rate of these samples, compared with OLSR-1. The effect is more important in OLSR-2 than in OLSR-3, due to the fact that the amine moiety in CN-PT attaches to Pt directly via a coordination bond, while N-PHMS does not. As a results, the curing rate of OLSR-2 is less than that of OLSR-3. OLSR-4 exhibits the lowest curing rate, possibly due to synergy between CN-PT and N-PHMS.

\subsubsection{Tensile bond strength analysis}

Variations in the tensile bond strengths of different OLSR coatings are shown in Fig. 12. The results indicate that CN-PT addition does not significantly affect the tensile bond strength of OLSR-2 (ca.0.26 $\mathrm{MPa}$ ), compared with OLSR-1 (ca.0.24 MPa). However, the incorporation of N-PHMS substantially enhances the bond strength of OLSR-3 (ca.1.26 MPa), compared with the former two samples. This effect may be attributed to the significantly improved surface tension and wetting properties of PCBs in silane solutions containing N-PHMS (see Sect. 3.4.3), as well as to the role of N-PHMS in promoting the chemical reaction between OLSR and the PCB surface. EDS analysis confirms that the interfacial reaction of OLSR with PCBs is mediated by secondary amide groups (see Sect. 3.4.4). Finally, the OLSR-4 sample containing both CN-PT and N-PHMS has the highest tensile strength (ca. $1.63 \mathrm{MPa}$ ), which suggests that CN-PT and N-PHMS exhibit synergistic effects. In addition, the bond strengths of OLSR-4 (ca. 1.63 MPa) was better than those of TL-GC02 (ca. 1.27 MPa).

\subsubsection{Surface tension analysis}

As shown in Fig. 13, OLSR-1 and OLSR-2 exhibit highly similar surface tension values (ca. 19.00 and $19.05 \mathrm{mN} / \mathrm{m}$, respectively). Thus, the addition of CN-PT does not significantly improve the wetting properties of the PCB board in OLSR, resulting in only minor variation in tensile bond strength. N-PHMS, on the other hand, has an appreciable effect on surface tension, as it increases the values corresponding to OLSR-3 and OLSR-4 to ca. 19.55 and $19.69 \mathrm{mN} / \mathrm{m}$, respectively, due to the addition of polar groups. This is consistent with the results of tensile bond strength analysis.

\subsubsection{SEM-EDS analysis}

Considering the similarity between OLSR-1 and OLSR-2 and between OLSR3 and OLSR-4, the surface morphologies of PCB samples coated with either OLSR-1 or OLSR-4 were analyzed by SEM-EDS. The FESEM images presented in Fig. 14 show that the OLSR-1-coated sample exhibits an almost pure interface failure with no remains on the coating surface. This indicates that the bonding strength between OLSR-1 and the PCB boards is poor. Meanwhile, interface and cohesive mixing failures are observed for the OLSR-4-coated sample, with abundant solid remains on the coating surface. Thus, OLSR-4 and the PCB 
boards are strongly bonded. The composition of the failure surface of the analyzed samples was assessed using EDS. The results illustrated in Fig. 15 show that the failure surface of OLSR-4 is comprised of $\mathrm{Ba}$ and $\mathrm{S}$ elements, in addition to the $\mathrm{Si}, \mathrm{O}$, and $\mathrm{C}$ elements available on the OLSR-1 failure surface. Si, $\mathrm{O}$, and $\mathrm{C}$ exist in the form of $\mathrm{Si}-\mathrm{O}-\mathrm{Si}, \mathrm{O}-\mathrm{Si}-\mathrm{O}$, or $\mathrm{Si}-\mathrm{C}$ groups in silane films, whereas the $\mathrm{Ba}$ and $S$ elements originate from the PCB surface. Compared with OSLR-1, the amount of Si on the failure surface of OLSR-4 is significantly decreased, while the amounts of $C$ and $O$ are relatively the same. These results indicate that the PCB boards are more strongly bonded to OLSR-4 than to OLSR-1, which is consistent with the bonding strength data.

\subsection{Application test}

\subsubsection{Salt-spray test analysis}

The corrosion protection effects of OLSR samples were assessed in a static mode using salt-spray tests. The results presented in Fig. 16 demonstrate that after 300 h, the OLSR-1 and OLSR-2 coatings of PCBs become detached due to adhesion bond damage. However, OLSR-3 and OLSR-4 remain strongly bonded to the PCB surfaces. Therefore, we may conclude that compared with CN-PT, N-PHMS is much more effective in enhancing the corrosion protection properties of OLSR.

\subsubsection{Continuous power-on test analysis}

The dynamic of corrosion protection properties of OLSR coatings was investigated via continuous poweron testing. The obtained results are illustrated in Fig. 17, and they show that the duration of continuous power-on in brine decreases in the following order: OLSR-4 > OLSR-3 > OLSR-2 > OLSR-1. This trend is attributed to the variation of bonding strengths of OLSR coating materials. Higher bonding strength leads to greater anti-corrosion protection efficiency. Considering that the corrosion protection properties of OLSR-4 are better than those of OLSR-3, CN-PT and N-PHMS may interact synergistically. In addition, the Continuous power-on times of OLSR-4 (2.32 h) was better than those of TL-GC02 (1.87 h).

\subsection{Possible mechanism of CN-PT/N-PHMS synergism}

As seen above, the storage stability and bond strength of OLSR coating are most effectively enhanced by combining CN-PT and N-PHMS. Thus, it is expected that these two entities may interact synergistically. Figures 18 and 19 present the proposed mechanism of synergistic CN-PT/N-PHMS effects in improving storage stability and corrosion resistance of OLSR coating, respectively. This mechanism suggests that unlike CN-PT (OLSR-2), the amide of N-PHMS does not undergo chemical bonding with the platinum catalyst, which leads to a weakened inhibition effect (OLSR-3). However, the hydrogen bonds formed between the secondary amide groups of different N-PHMS molecules allow for indirect bonding between $\mathrm{N}-\mathrm{PHMS}$ and the platinum catalyst, resulting in the depression of the curing rate at higher N-PHMS content. When CN-PT and N-PHMS are added together, hydrogen bonds are formed between the secondary amide of N-PHMS and the primary amide of CN-PT, resulting in a significantly improved interface of OLSR adhesion on PCBs. This enhances the bond strength and corrosion resistance of the OLSR coating. 


\section{Conclusions}

In summary, OLSR coating featuring outstanding storage stability and bond strength was successfully prepared by incorporating CN-PT and N-PHMS. Based on viscosity tests, the concentration of $6 \%$ was taken to be the optimum concentration of acrylonitrile, with the ability to store the material for more than $180 \mathrm{~d}$ at $25^{\circ} \mathrm{C}$ and cure it with PCB within $2 \mathrm{~h}$ at $80^{\circ} \mathrm{C}$. As for N-PHMS, it enhances the bond strength of OLSR coating by increasing the surface tension of the material and promoting the interfacial reaction of reactive groups. Tensile strength and Shore $A$ hardness assessments indicate that the optimum content of N-PHMS is 3\%. The combination of CN-PT and N-PHMS synergistically reduces the curing rate and further enhances storage stability via hydrogen bonding on the PCB surface. It also could significantly improved the salt-spray resistance and continuous power-on performance of OLSR coating. Our findings exhibited great potentials for fabricating OLSR coating with excellent long-term stable service performance including high storage stability and protective ability. But the application of OLSR coating will be limited in occasion when the heating is not allowed.

\section{Declarations}

\section{Acknowledgments}

This research did not receive any specific grant from funding agencies in the public, commercial, or notfor-profit sectors.

\section{Competing interests}

The authors declare that they have no known competing financial interests or personal relationships that could have appeared to influence the work reported in this paper.

\section{References}

1. C. Chen, Y. He, C. Liu, H. Xie, W. Yu, Comprehensive excellent performance for silicone-based

2. thermal interface materials, through the synergistic effect between graphene and spherical alumina, Journal of Materials

3. Science: Materials in Electronics. 31 (2) (2020) 4642-4649

4. https://link.springer.com/article/10.1007/s10854-020-03016-3

5. Q. Chen, B. Xi, J. Zhang, X. Wang, H. Yang, Dielectric and space charge characteristics of

6. nano-modified liquid, silicone rubber for high-voltage DC cable accessories, Journal of Materials Science: Materials in

7. Electronics. 31 (8) (2020) 16819-16829

8. https://link.springer.com/article/10.1007/s10854-020-04236-3 
9. E. Salahinejad, R. Eslami-Farsani, L. Tayebi, Corrosion failure analysis of printed circuit boards exposed to H2S-containing

10. humid environments, Engineering Failure Analysis. 79(9), 538-546 (2017)

11. https://

12. L.K. Namitha, S. Ananthakumar, M.T. Sebastian, Aluminum nitride filled flexible silicone rubber composites for

13. microwave substrate applications, Journal of Materials Science: Materials in Electronics. 26(11), 891-897 (2015)

14. https://link.springer.com/article/10.1007/s10854-014-2479-9

15. K.-H. Shin, H.A.D. Nguyen, J. Park, D. Shin, Dongjin Lee, Surface analysis of silver-plated circuit

16. boards in a salt-spray environment, Journal of Coatings Technology and Research. 14(10), 95-106 (2017)

17. $h$ ttps://link.springer.com/article/10.1007/s11998-016-9844-y

18. Qian Wang, Shuye Zhang, Guiming Liu, Tiesong Lin, PengHe, The mixture of silver nanowires and nanosilver-coated

19. copper micronflakes for electrically conductive adhesives to achieve high electrical conductivity with low percolation threshold, Journal of Alloys and Compounds. 820(4)(2020) 153184

20. https://www.sciencedirect.com/science/article/abs/pii/S0925838819344305

21. Qian Wang, Shuye Zhang, Tiesong Lin, Pengzhe Zhang, Peng He,Kyung-Wook Paik, Highly mechanical and

22. high-temperature properties of Cu-Cu joints using citrate-coated nanosized Ag paste in air, Progress in Natural Science:

23. Materials International.9(1)(2021)

24. https://www.sciencedirect.com/science/article/pii/S1002007120305797

25. Michele T. Byrne, Yurii K. Gun'ko, Recent Advances in Research on Carbon Nanotube-Polymer Composites, Advanced

26. Materials. 22(15)(2010)1672-1688

27. https://onlinelibrary.wiley.com/doi/abs/10.1002/adma.200901545

28. A. Latifi, M. Imani, M.T. Khorasani, M.D. Joupari, Plasma surface oxidation of 316L stainless steel for improving adhesion

29. strength of silicone rubber coating to metal substrate, Appl. Surf. Sci. 320(11), 471-481 (2014)

30. https://

31. J. Shan Cao, Y. Wang, D. Zhang, Chen, The effectiveness of an antifouling compound coating based on a

32. silicone elastomer and, colored phosphor powder against Navicula species diatom, Journal of Coatings Technology and 
33. Research. 10 (12) (2013) 397-406

34. https://link.springer.com/article/10.1007/s11998-012-9457-z

35. M. Qipeng Zhang, Wu, Structure of vinyl polysiloxane on properties of polyacrylates film and its pigment printing

36. application .Journal of Coatings Technology and Research.17 (11) (2020) 937-948

37. https://link.springer.com/article/10.1007/s11998-020-00320-9

38. X. Chengyu Ju, R. Wang, X. Zhu, Ren, Comparative Analysis of Printed Circuit Board Coating on Corrosion

39. Test, Engineering Asset Management - Systems. Professional Practices and Certification 30(12), 1359-1369 (2014)

40. https://link.springer.com/chapter/10.1007\%2F978-3-319-09507-3_115

41. U. Eduok, O. Faye, J. Szpunar, Recent developments and applications of protective silicone coatings: A review of PDMS

42. functional materials, Progress in Organic Coatings. 111(10), 124-163 (2017)

43. https://www.sciencedirect.com/science/article/pii/S0300944016306464

44. Y.P. Li, X.R. Zeng, X.J. Lai, H.Q. Li, W.Z. Fang, Effect of the platinum catalyst content on the tracking and erosion

45. resistance of addition-cure liquid silicone rubber, Polym. Testing 63(10), 92-100 (2017)

46. https://

47. T. Liu, X.R. Zeng, W.Z. Fang, X.J. Lai, H.Q. Li, Synthesis of a novel hydantoin-containing silane and its effect on the

48. tracking and bacteria resistance of addition-cure liquid silicone rubber, Applied Surface Science. 423 (11) (2017) 630-640

49. https://

50. C.X. Xie, X.R. Zeng, W.Z. Fang, X.J. Lai, H.Q. Li, Effect of alkyl-disubstituted ureido silanes with different alkyl chain

\section{Figures}


$\mathrm{H}_{2} \mathrm{PlCl}_{6}\left(\mathrm{H}_{2} \mathrm{C}=\mathrm{CH}-\left.\right|_{\mathrm{Si}} ^{\mathrm{CH}_{3}}-\mathrm{O}-\left.\right|_{\mathrm{Ci}} ^{\mathrm{CH}_{3}}-\mathrm{C}=\mathrm{CH}_{3}\right)_{2}$
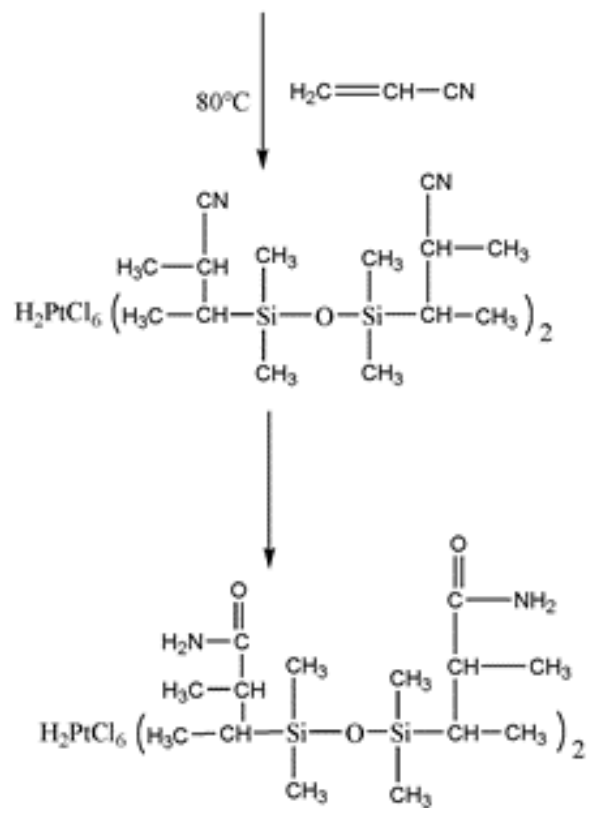

\section{Figure 1}

CN-PT synthesis via thermal polymerization reaction.
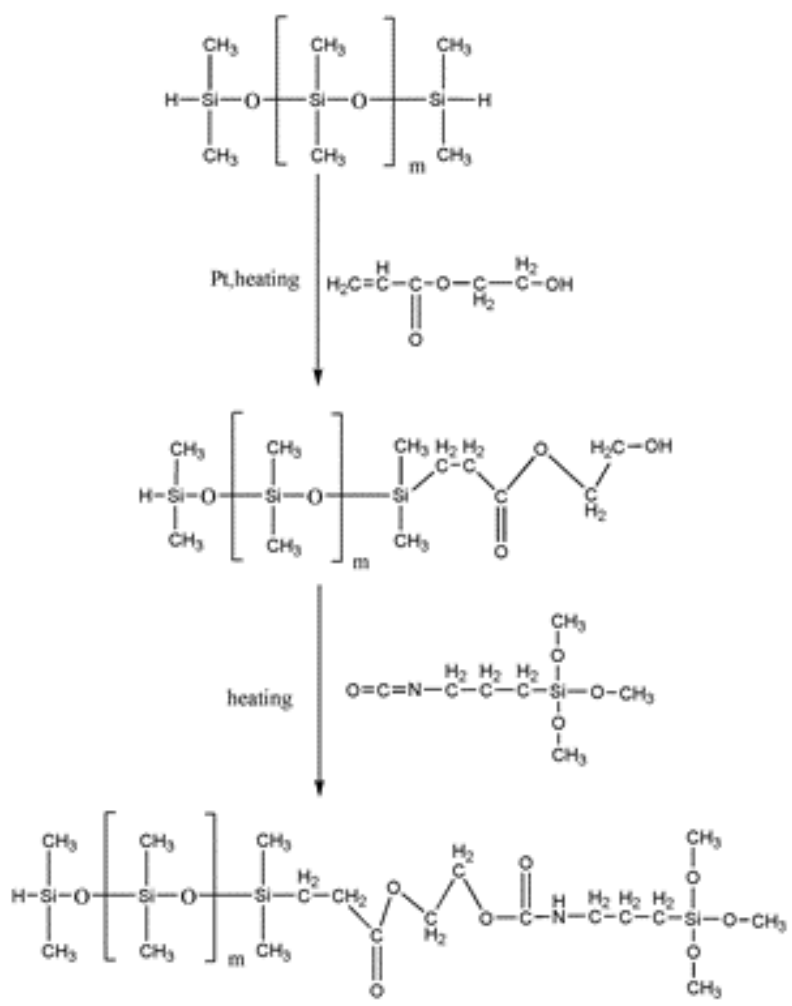

Figure 2 
N-PHMS synthesis via a two-step hydrosilylation reaction.

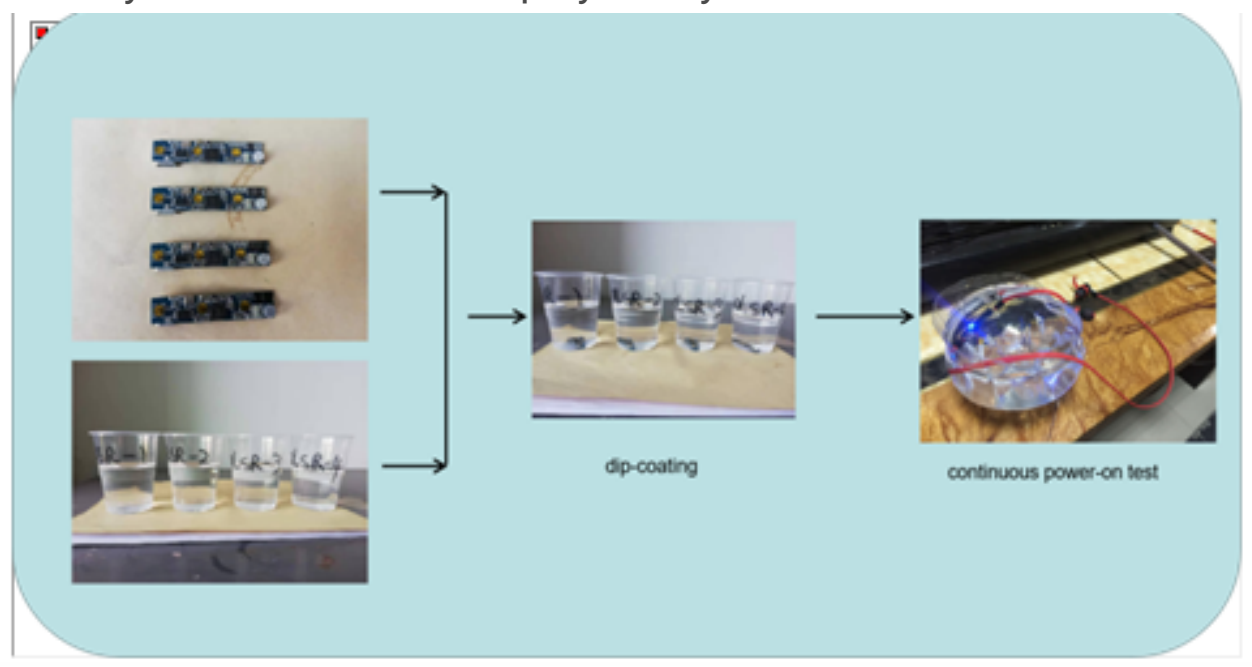

Figure 3

Schematic of continuous power-on testing.

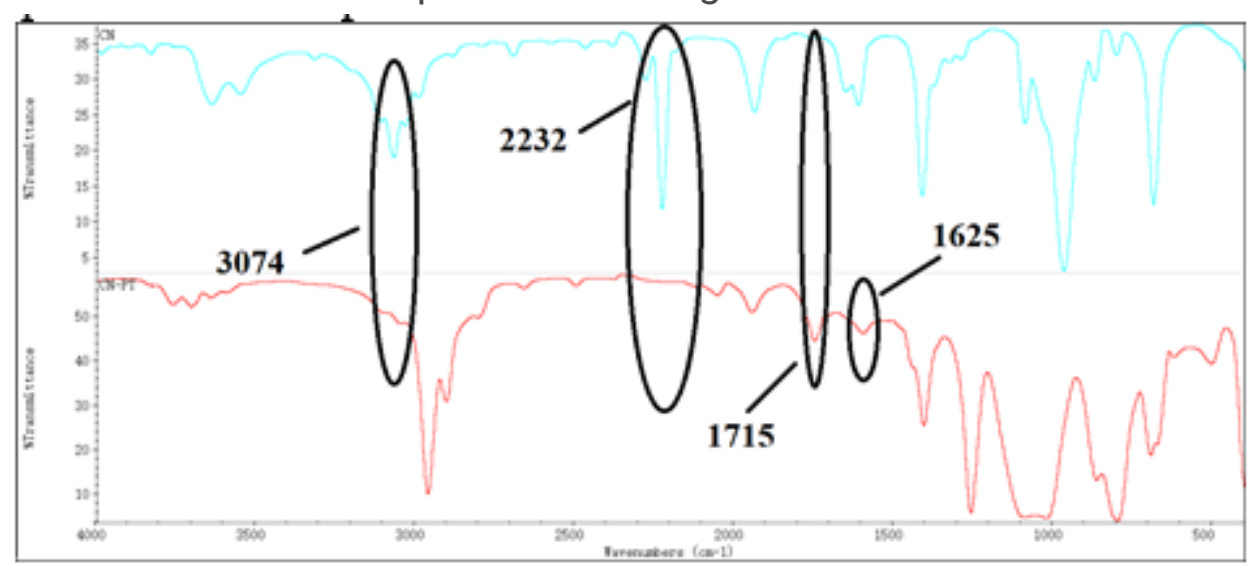

Figure 4

ATR-FTIR spectra of CN, and CN-PT.

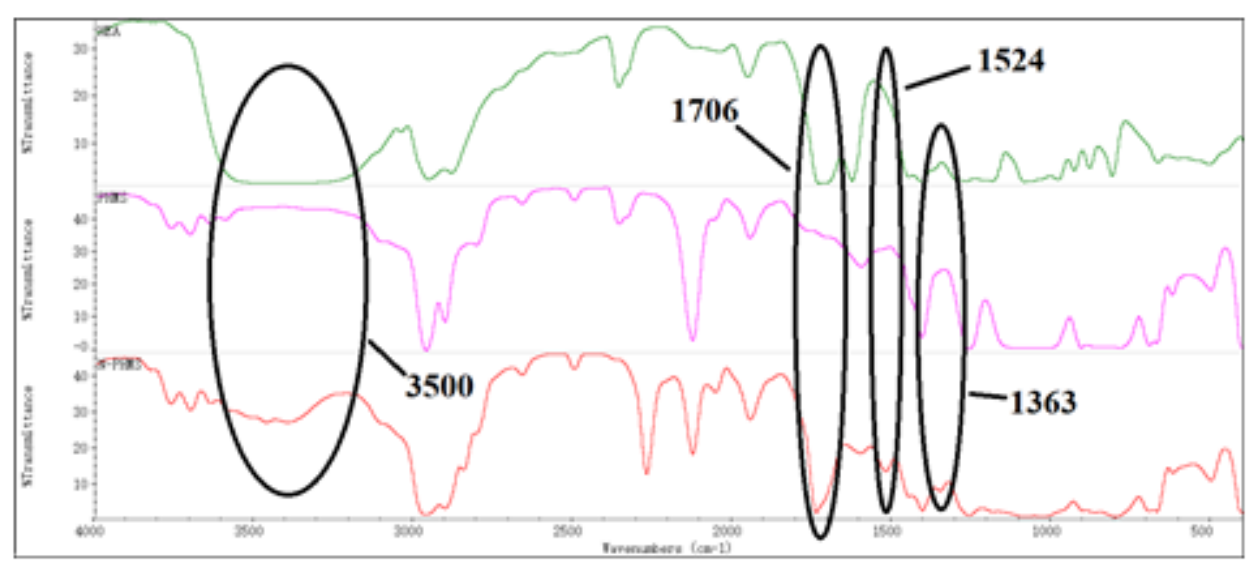

Figure 5 
ATR-FTIR spectra of PHMS and N-PHMS.

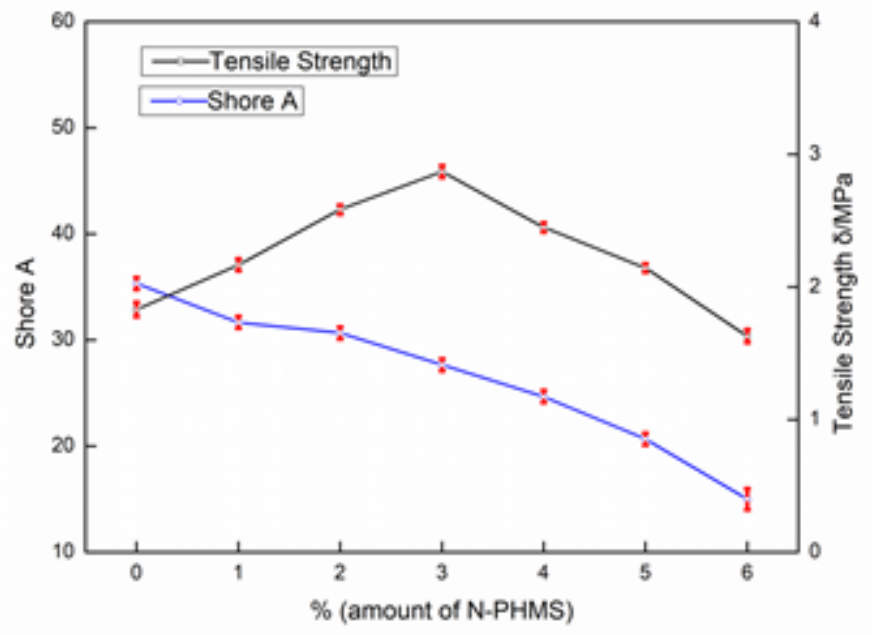

\section{Figure 6}

Tensile strength and Shore A hardness of OLSR coating materials prepared using different amounts of $\mathrm{N}$ PHMS.

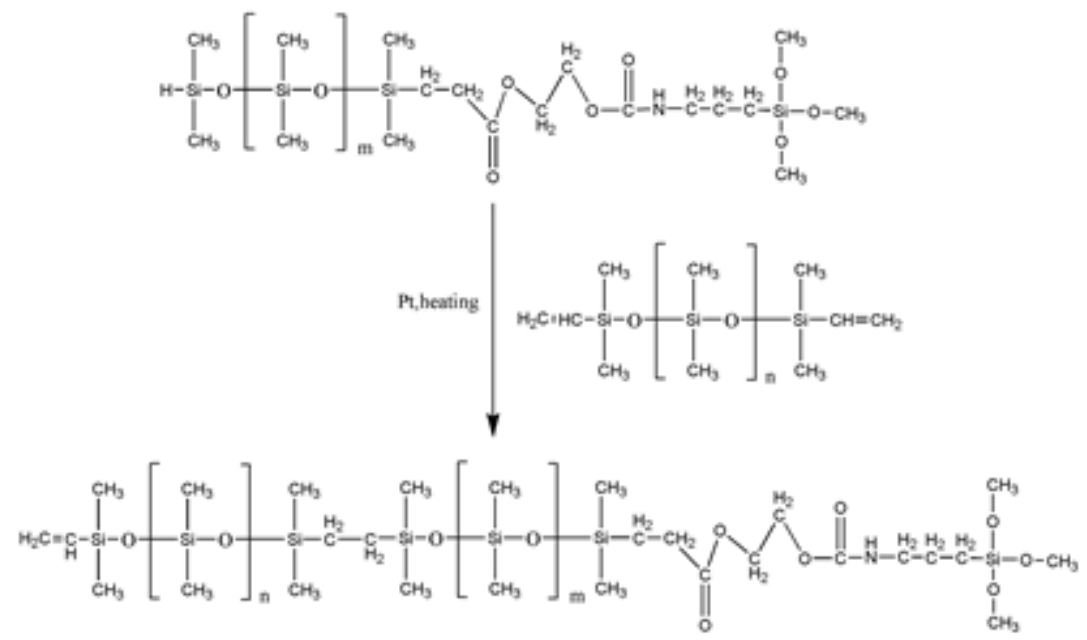

\section{Figure 7}

The schematic diagram of the reaction of the N-PHMS and VPDMS 


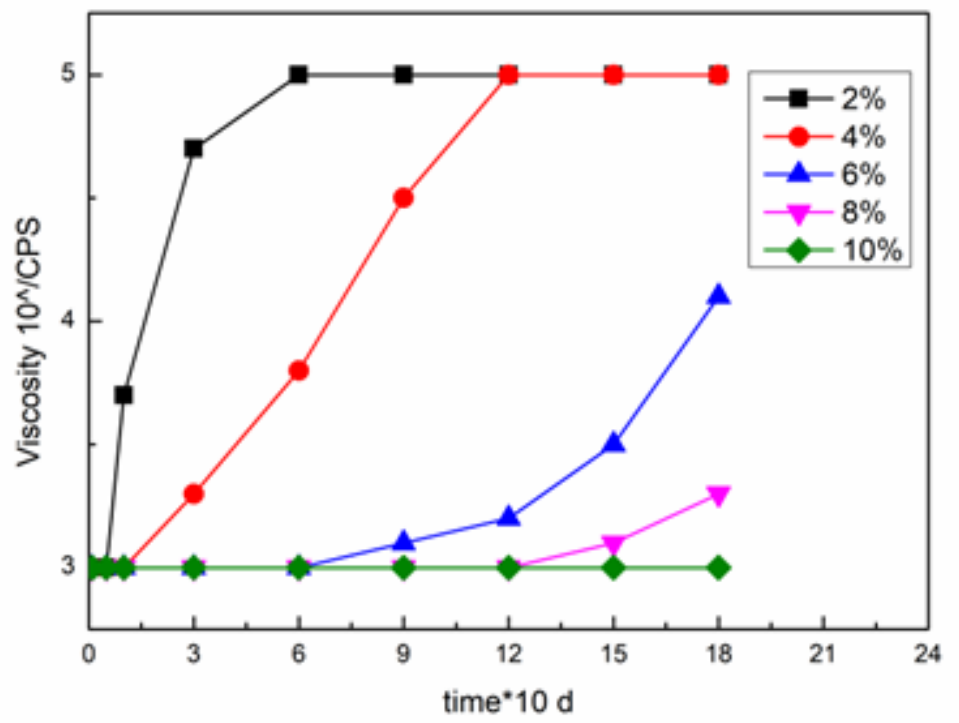

Figure 8

Temporal evolution of OLSR viscosity at $25^{\circ} \mathrm{C}$ (different acrylonitrile concentrations in CN-PT).

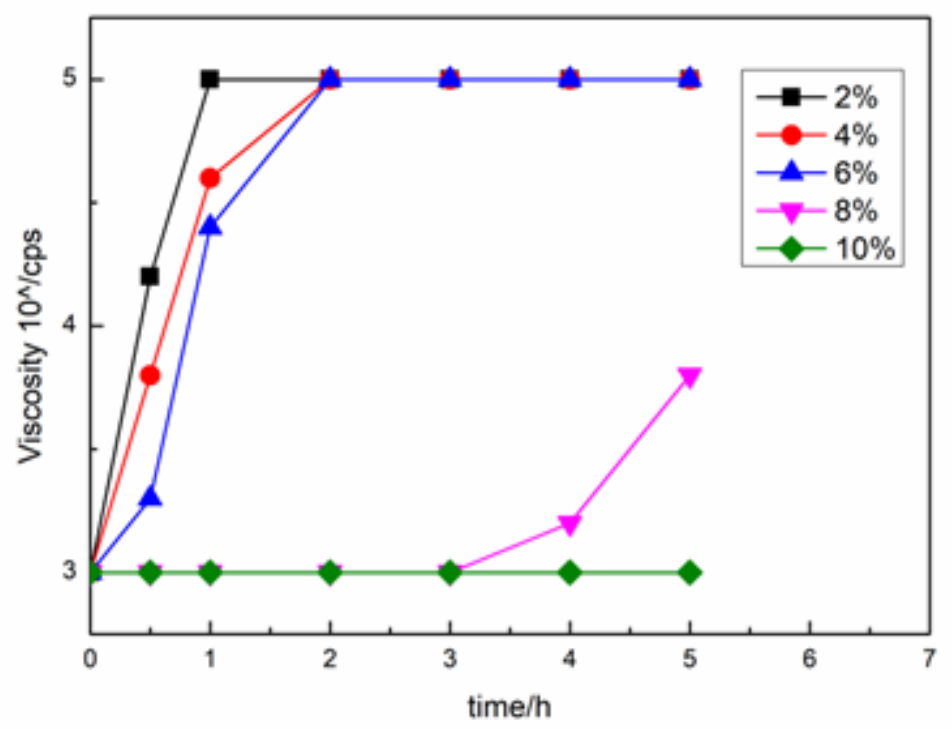

Figure 9

Temporal evolution of OLSR viscosity at $80^{\circ} \mathrm{C}$ (different acrylonitrile concentrations in CN-PT). 


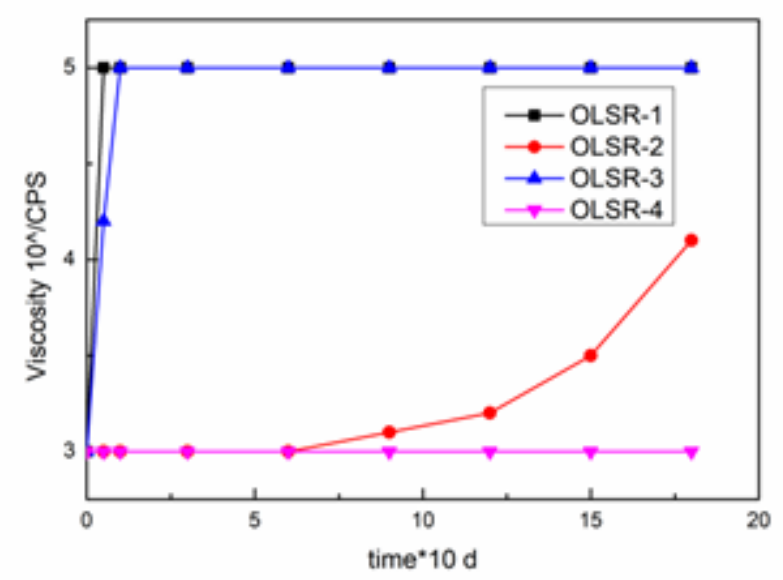

Figure 10

Temporal evolution of the viscosity of different OLSR samples at $25^{\circ} \mathrm{C}$.

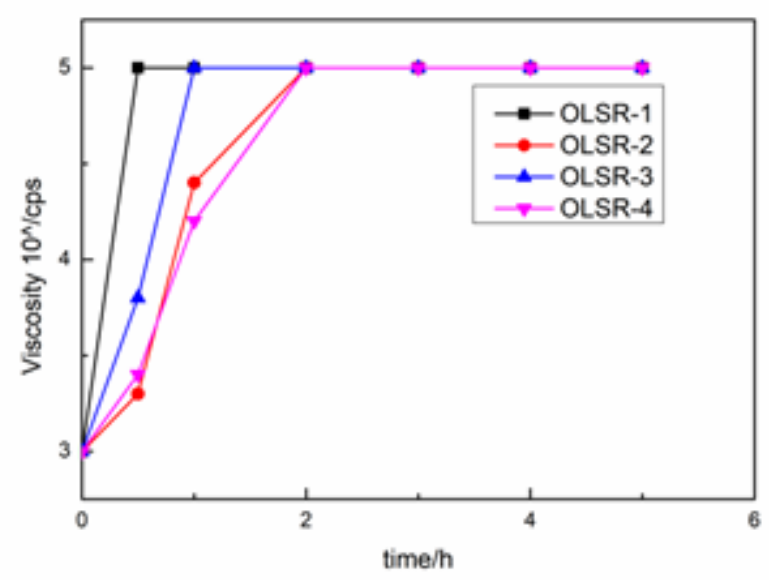

Figure 11

Temporal evolution of the viscosity of different OLSR samples at $80^{\circ} \mathrm{C}$.

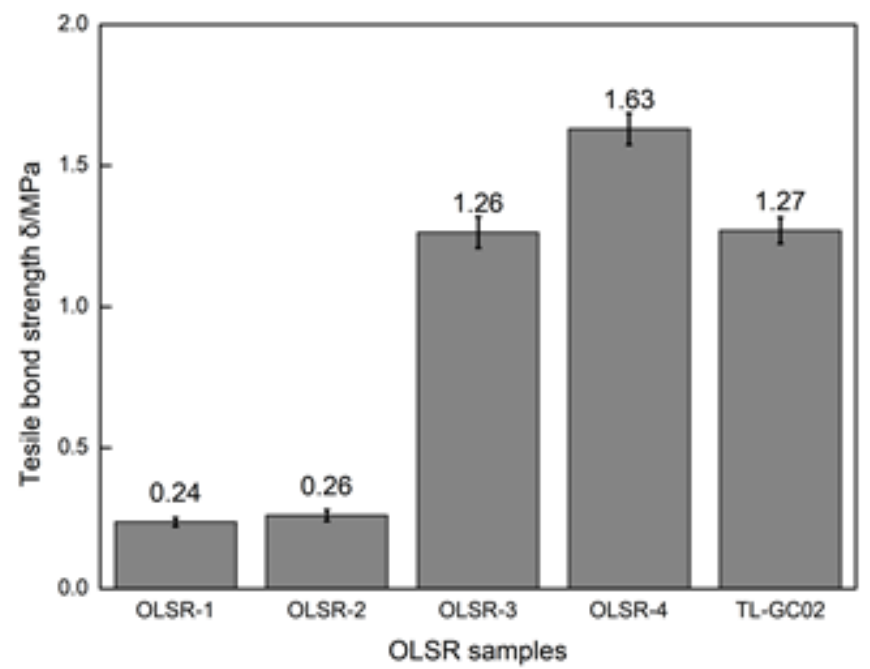

Page $17 / 21$ 
Figure 12

Bond strengths of different OLSR samples.

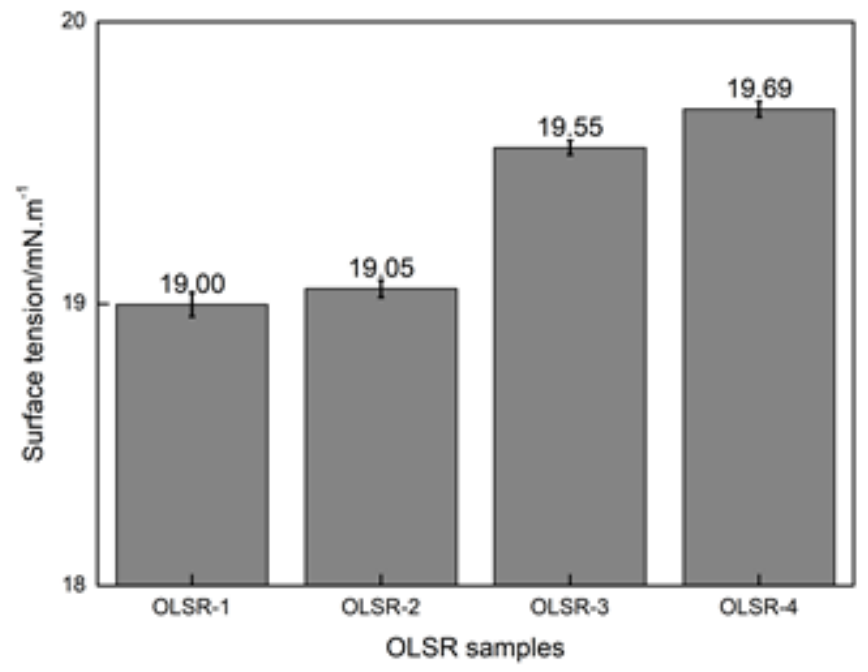

Figure 13

Surface tension of different OLSR samples.
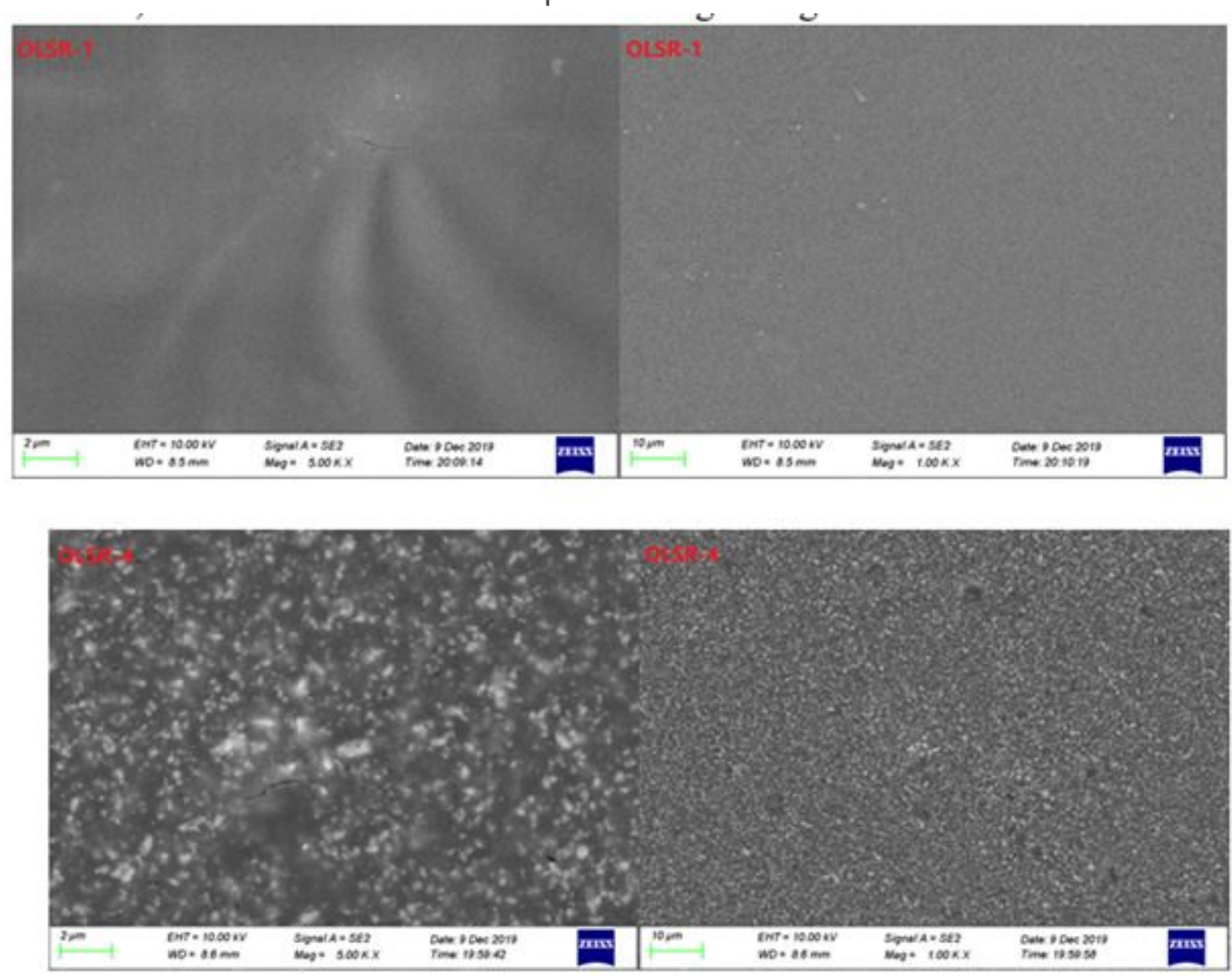

Figure 14 
FE-SEM micrographs of the OLSR surface after shear test: (a, b) OLSR-1 and (c, d) OLSR-4.

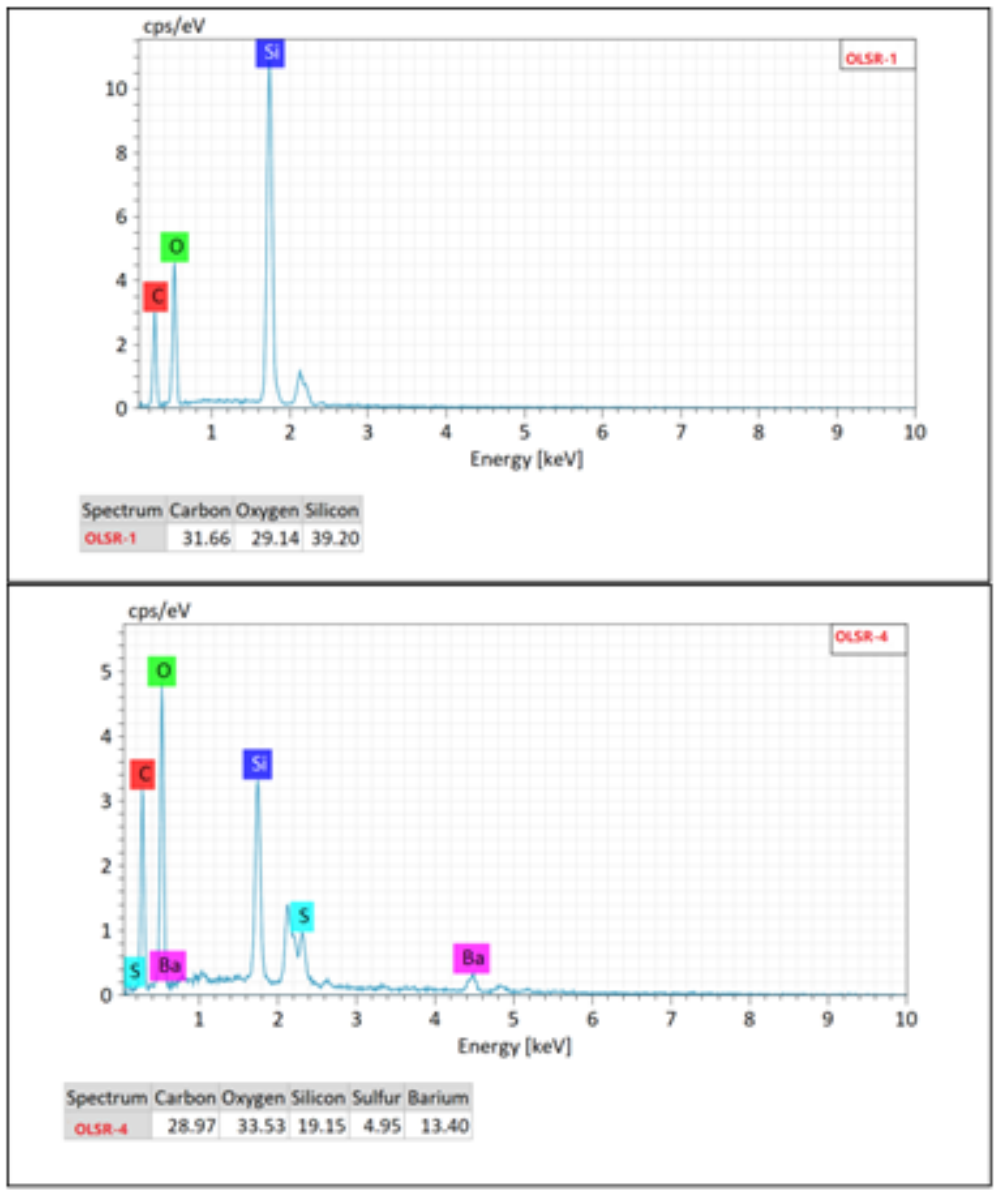

\section{Figure 15}

EDS analysis of the OLSR surface (the mass proportions) after shear test: (a) OLSR-1 and (b) OLSR-4. 

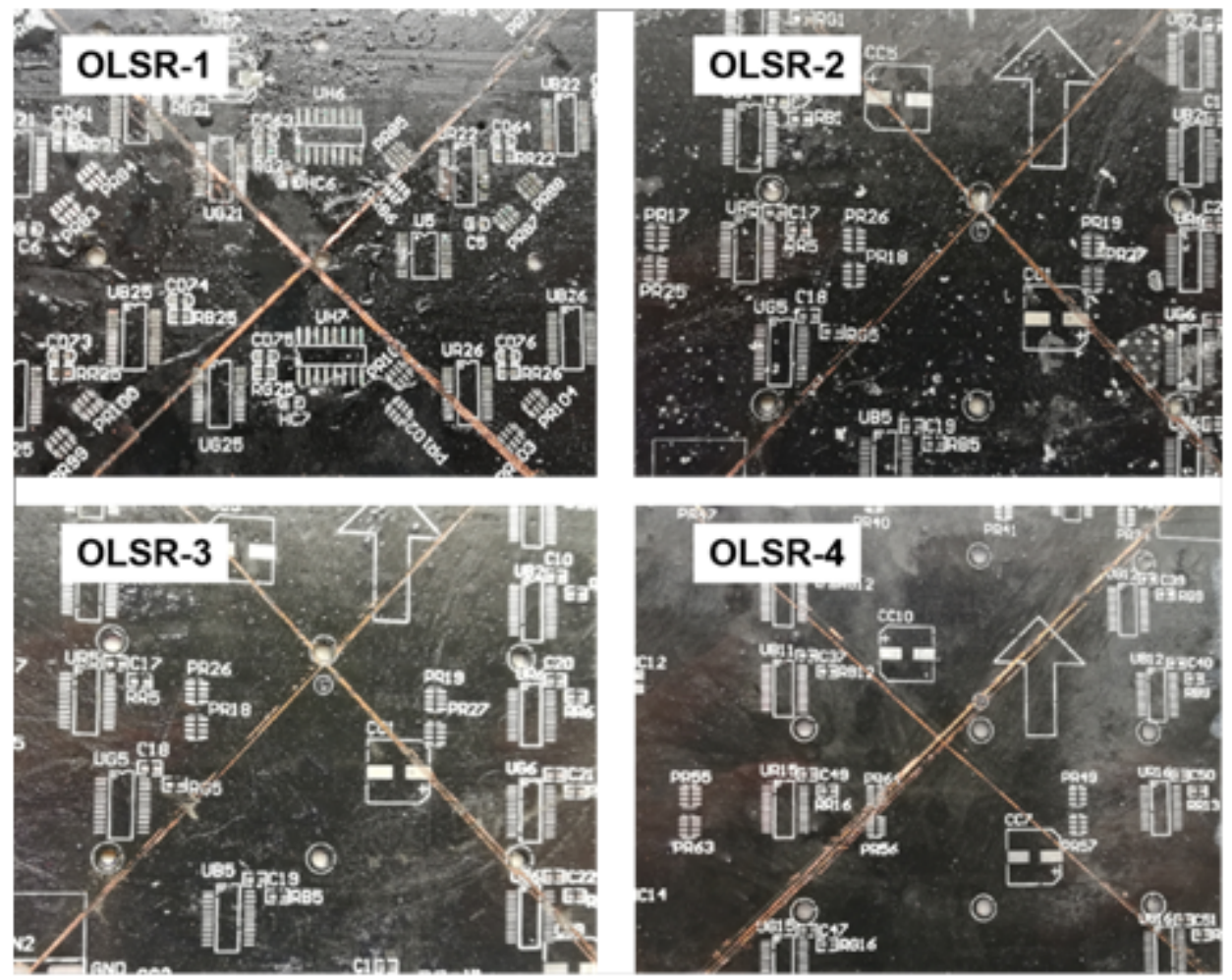

Figure 16

Salt-spray tests $(300 \mathrm{~h})$ performed on different OLSR-coated PCB samples.

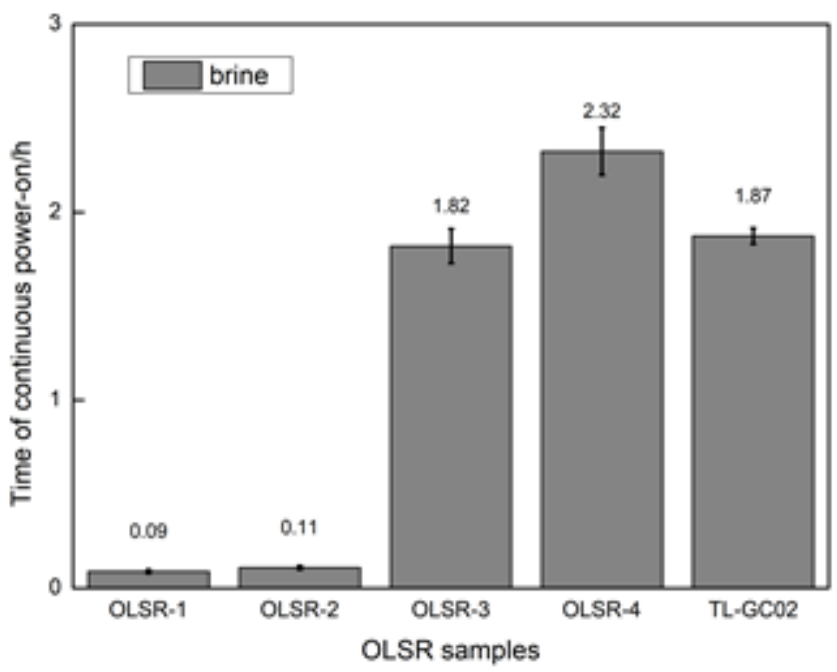

Figure 17

Continuous power-on times of different OLSR coating materials. 


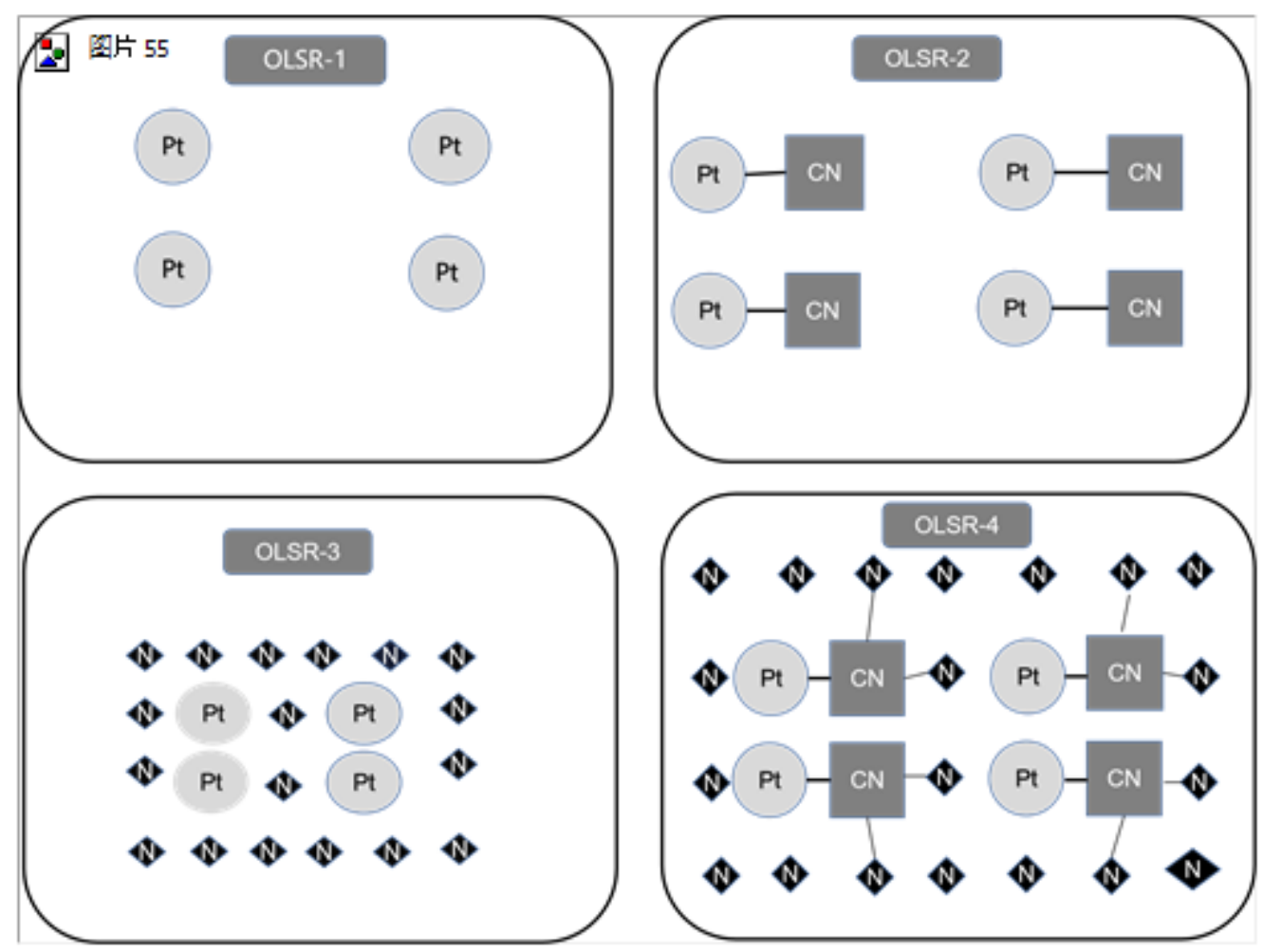

Figure 18

Possible mechanism of CN-PT and N-PHMS synergistic effects in enhancing the storage stability of OLSR coating.

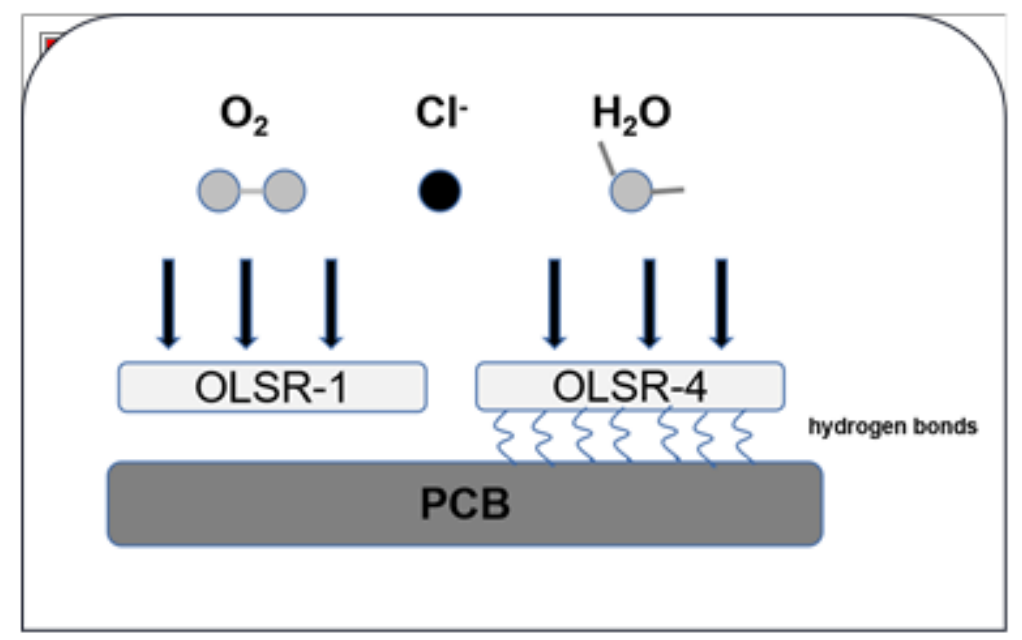

Figure 19

Possible mechanism of CN-PT and N-PHMS synergistic effects in enhancing the corrosion resistance of OLSR coating. 